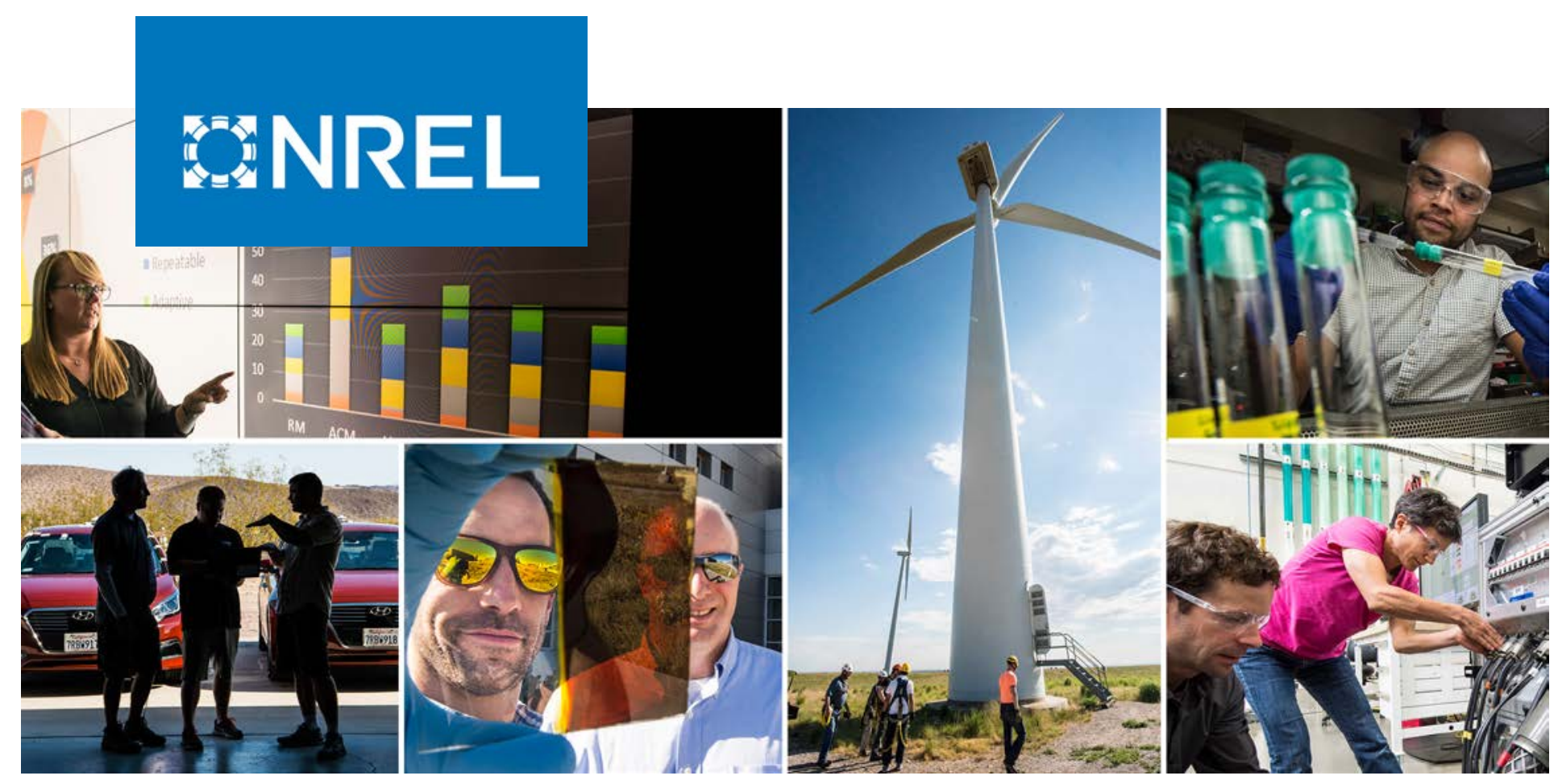

\title{
Modeling Needs for Power Semiconductor Devices and Power Electronics Systems
}

\section{Preprint}

Ramchandra Kotecha, Gilberto Moreno, Barry Mather, and Sreekant Narumanchi

\section{National Renewable Energy Laboratory}

Presented at 2019 IEEE International Electron Devices Meeting (IEDM) San Francisco, California

December 7-11, 2019

NREL is a national laboratory of the U.S. Department of Energy Office of Energy Efficiency \& Renewable Energy

Operated by the Alliance for Sustainable Energy, LLC

This report is available at no cost from the National Renewable Energy Laboratory (NREL) at www.nrel.gov/publications.

\section{Conference Paper}

NREL/CP-5400-74666

February 2020 


\title{
GNREL
}

\section{Modeling Needs for Power Semiconductor Devices and Power Electronics Systems}

\section{Preprint}

\author{
Ramchandra Kotecha, Gilberto Moreno, Barry Mather, and \\ Sreekant Narumanchi
}

National Renewable Energy Laboratory

\author{
Suggested Citation \\ Kotecha, Ramchandra, Gilberto Moreno, Barry Mather, and Sreekant Narumanchi. 2020. \\ Modeling Needs for Power Semiconductor Devices and Power Electronics Systems: \\ Preprint. Golden, CO: National Renewable Energy Laboratory. NREL/CP-5400-74666. \\ https://www.nrel.gov/docs/fy20osti/74666.pdf.
}

\begin{abstract}
(c) 2020 IEEE. Personal use of this material is permitted. Permission from IEEE must be obtained for all other uses, in any current or future media, including reprinting/republishing this material for advertising or promotional purposes, creating new collective works, for resale or redistribution to servers or lists, or reuse of any copyrighted component of this work in other works.
\end{abstract}

NREL is a national laboratory of the U.S. Department of Energy Office of Energy Efficiency \& Renewable Energy Operated by the Alliance for Sustainable Energy, LLC

This report is available at no cost from the National Renewable Energy Laboratory (NREL) at www.nrel.gov/publications.

Contract No. DE-AC36-08GO28308
Conference Paper

NREL/CP-5400-74666

February 2020

National Renewable Energy Laboratory 15013 Denver West Parkway Golden, CO 80401

303-275-3000 • www.nrel.gov 


\section{NOTICE}

This work was authored by the National Renewable Energy Laboratory (NREL), operated by Alliance for Sustainable Energy, LLC, for the U.S. Department of Energy (DOE) under Contract No. DE-AC36-08GO28308. This work was supported by the Laboratory Directed Research and Development (LDRD) Program at NREL. The views expressed in the article do not necessarily represent the views of the DOE or the U.S. Government. The U.S. Government retains and the publisher, by accepting the article for publication, acknowledges that the U.S. Government retains a nonexclusive, paid-up, irrevocable, worldwide license to publish or reproduce the published form of this work, or allow others to do so, for U.S. Government purposes.

This report is available at no cost from the National Renewable Energy Laboratory (NREL) at www.nrel.gov/publications.

U.S. Department of Energy (DOE) reports produced after 1991 and a growing number of pre-1991 documents are available free via www.OSTI.gov.

Cover Photos by Dennis Schroeder: (clockwise, left to right) NREL 51934, NREL 45897, NREL 42160, NREL 45891, NREL 48097, NREL 46526.

NREL prints on paper that contains recycled content. 


\title{
Modeling Needs for Power Semiconductor Devices and Power Electronics Systems
}

\author{
Ramchandra Kotecha, Gilberto Moreno, Barry Mather, and Sreekant Narumanchi \\ National Renewable Energy Laboratory, Golden, CO, email: Ramchandra.Kotecha@nrel.gov
}

\begin{abstract}
As energy systems move towards wide-spread electrification, penetration of power semiconductor devices and power electronics continue to grow at a rapid pace. This paper attempts at reviewing the state-of-art in power semiconductor modeling and the existing state of modeling in general when in it comes to design of devices and systems. The modeling needs for semiconductor devices vary depending on the end-goal and the level of abstraction needed towards model formulation also changes with the size and complexity of system to be designed. Finally, key aspects of thermal design and modeling for power electronics is also discussed.
\end{abstract}

\section{INTRODUCTION}

Power Electronics systems are increasingly becoming prevalent in the changing landscape for modern energy systems [1]. At the heart of power electronics systems are power semiconductor devices that enable the energy conversion from source side to the loads. The modeling field in power semiconductor devices has not been fully matured when compared with analog and digital semiconductor devices and is still evolving to adapt to recent disruptions in power electronics caused by the penetration of wide band-gap devices (WBG) [2][5]. The major challenges being faced towards modeling of power semiconductor devices stems from the fact that power electronic systems are very diverse and the complexities very from a simple dc-dc energy conversion topology to a complex system such as a grid-tied power electronics interface or a standalone microgrid for distributed generation. In such cases, it becomes extremely difficult to standardize on the modeling approaches for power semiconductor devices.

\section{MODELING NeEDS FOR POWER SEMICONDUCTOR DEVICES AND EMERGING WIDE BANDGAP DEVICES}

\section{A. Device Design Needs}

Wide bandgap devices have come a long way in power electronics since the first $\mathrm{SiC}$ devices were demonstrated. The process development and fabrication of power semiconductor devices for new material is an extremely challenging task due to the lack of information on characterization of material and its performance. Finite element modeling in semiconductor devices has largely become possible due to commercial technology computer aided design (TCAD) tools such as Sentaurus and ATLAS Silvaco [7]. There are some open source TCAD tools like DEVSIM also available for device design. These tools are mainly based on the solutions of carrier transport equations as a function of lattice temperature. Regardless, TCAD modeling is highly suitable for process and device design and may not be suitable for circuit or system simulations. Fig. 5 shows the results obtained for a TCAD model developed for $\mathrm{Ga}_{2} \mathrm{O}_{3}$ devices

\section{B. Modeling for Circuit Design and System Simulation}

While finite-element models are an attractive choice for device design, they are practically impossible to use for circuit and system design. The modeling needs for circuit design require that the semiconductor models are in their compact form and represent 1-D representation of the higher-order effects. Also, the models should be parameterized in a way that the parameters can be easily extracted from the measured device characteristics. The key device characteristics such as dc $I V, C V$, gate charge, and thermal impendence are usually needed to characterize a model suitable for circuit and system design simulations.

Semiconductor models represent different levels of abstraction depending on the end-goal (Fig.1 and 2). E.g., if the goal is to evaluate a device just for its switching characteristics, a compact model with detailed channel current and charge equations must be used such that the parameters of the model can be easily extracted from the measured device characteristics as shown in Fig. 3. A detailed model which is physics-inspired is likely to capture the overall switching and conduction losses and is also more likely to accurately predict the frequency response as well as time-domain behavior resulting from interactions between device and circuit parasitics (Fig.4). However, a wide range of power electronic systems are now way more complex than a simple switching converter. A lot of system level designs require simulation engines like MATLAB/Simulink ${ }^{\circledR}$ and PLECS. A complex switching device model may consume a lot of computing resources when used in complex system-level designs for simulations. As a result, tools like MATLAB/Simulink ${ }^{\circledR}$ and PLECS have become very popular wherein the semiconductor switches, although not ideal, do not represent the same level of complexities as behavioral or physics-inspired models would represent [8].

As a result, modeling needs continue to evolve and adapt to the changing landscape of evolving energy systems. The complexity of any given model largely depends on the level of abstraction made from its actual physical behavior. And the levels of abstraction are usually determined depending on the end-goal of the model. The higher the level of abstraction, more physical in nature the model is. Thus, the choice of complexity of the model is need-based and the availability of software tools as well as computing resources. A complex model is likely to consume more computing resources and simulation time. Thus, 
there is always a trade-off between speed and desired accuracy of the model. Ideally, a model which produces the desired outcome with least amount of computing resources and time is more useful regardless of the level of complexity and abstraction. Although, parameterization of the model is also an important factor to consider wherein 1-D physics-inspired models may provide ease of parameterization when compared with semi-empirical or behavioral models. Purely behavioral models may be more useful when supplied with fitting software tools designed with fast optimization algorithms. Historically, semiconductor device models in Berkeley SPICE were classified as shown in Table 1. This standardization first came into being when SPICE (Simulation Program with Integrated Circuit Emphasis) was introduced and developed by UC Berkeley. Later, UC Berkeley introduced BSIM models for MOSFET devices [5]. The need for standardization stemmed from the fact that integrated circuit (IC) design was a rapidly growing field, and the need for compact models was imminent due to the large volumes of demands for ICs for analog and digital electronics applications. The major driving force for this demand was rapid growth in chip manufacturing companies like Intel, IBM, HP, and TI.

Table 1: Different Levels of SPICE MOSFET model

\begin{tabular}{|cc|}
$\begin{array}{c}\text { Level of } \\
\text { Abstraction }\end{array}$ & $\begin{array}{c}\text { Important Device Behavior Captured at } \\
\text { Each Level }\end{array}$ \\
\hline Level 1 & Basic IV behavior for short-channel devices \\
\hline Level 2 & $\begin{array}{c}\text { Body effect, field dependent electron } \\
\text { mobility, channel length modulation, carrier } \\
\text { velocity saturation }\end{array}$ \\
\hline
\end{tabular}

Level 3 Added parameters beyond Level 1 and 2 models for empirical fitting

Later, BSIM models were rolled out by Berkeley group to overcome the limitations of early generations of SPICE models in integrated circuit design. BSIM models have close to 200 parameters and are standardized for many commercial simulators that supports its models implemented in $C$ or Verilog-A. The models are physics-based, and the parameters may be extracted directly from the measured device characteristics and the models are scalable to process and design parameters. In general, the accuracy and fidelity of BSIM transistor models has vastly improved the quality and productivity of IC design based on silicon (Si) devices. The standardization process of these models is guided by compact model coalition (CMC) that continuously monitors the advances and needs for advanced nodes in Si processes.

However, when it comes to power semiconductor devices, this kind of standardization does not seem to exist. Various software programs provide semiconductor switch models for power electronics design and simulation that blends with their simulation engines. The models are mostly behavioral or subcircuit-based and are usually not scalable to physical and process parameters. Recently, OnSemi has started to roll out SPICE models for its power device products that are claimed to be physically scalable and directly tied to the process device parameters [6]. The power MOSFET model captures device electro-thermal behavior with cauer impedance network that calculates device junction temperature $\left(T_{j}\right)$ to characterize the effect of device self-heating on device transient performance. The model is demonstrated as scalable to process parameters. The channel current is modeled by BSIM3 model equations which requires further research because the device structure presented in the model is representative of a VDMOS device while the BSIM models are primarily for low voltage lateral MOSFET devices used in IC designs. Nevertheless, the effort to standardize modeling approaches for power semiconductor devices is in the right direction considering the challenges posed by emerging WBG devices.

\section{Thermal Modeling in Power Electronics}

Most automotive power electronics are cooled using a liquid (water-ethylene glycol solution) heat exchangers (Fig. 6). Some of the early inverter designs used a cold plate cooled configuration that mounted the power module to an aluminum cold plate with thermal grease at the interface. More recent inverter-designs now use a baseplate cooled configuration which eliminates the thermal grease (largest thermal resistance component) and directly cool the baseplate. Double side cooled modules are also an effective method to further improve thermal performance by removing heat from both sides of the module. The double side cooled approach is becoming more common with the advent of planar package designs that eliminate the wire bonds. Designing the convective cooling systems requires knowledge of the operating conditions. Computation fluid dynamics (CFD) methods can be used to design the fluid flow path and surface enhancement features (e.g., fins, coatings). Finite element (FE) methods can be used to compute the junction temperatures using the CFD-estimated convective heat transfer coefficients. Steady-state and transient thermal simulations can be conducted using the simpler FE models to evaluate various operating conditions.

\section{REFERENCES}

[1] Inna, "Advanced Power Electronics Will Rule Future Power Systems," INMR : Independent T\&D Information Resources, 22-Jun-2019.

[2] "Device Modeling - an overview | ScienceDirect Topics." [Online]. Available:https://www.sciencedirect.com/topics/materialsscience/device-modeling. [Accessed: 31-Jul-2019].

[3] D. L. Fuller, "Introduction to Modeling MOSFETS in SPICE," Microelectronic Engineering, p. 57, 2019.

[4] M. S. Hasan, M. S. A. Shawkat, S. Amer, S. K. Islam, N. McFarlane, and G. S. Rose, "Modeling Emerging Semiconductor Devices for Circuit Simulation," Modeling and Simulation in Engineering, May 2019.

[5] Y. S. Chauhan et al., "BSIM - Industry standard compact MOSFET models," in 2012 Proceedings of the European Solid-State Device Research Conference (ESSDERC), 2012, pp. 46-49.

[6] “ON Semiconductor: SPICE Modeling." [Online]. Available: https://www.onsemi.com/PowerSolutions/content.do?id=19077. [Accessed: 13-Aug-2019].

[7] "TCAD." [Online]. Available: https://www.synopsys.com/silicon/tcad.html. [Accessed: 13-Aug2019].

[8] "Learn with MATLAB and Simulink Tutorials." [Online]. Available: https://www.mathworks.com/support/learn-with-matlab-tutorials.html. [Accessed: 13-Aug-2019]. 


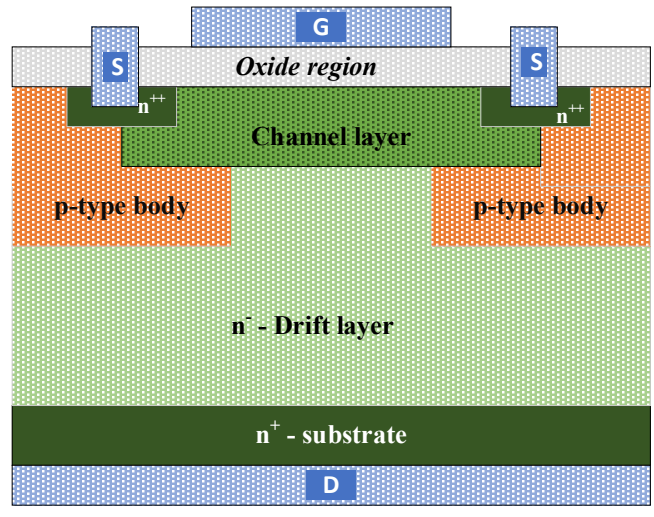

Fig. 1. (a) Representative 2-D device structure of a vertical power MOSFET device.

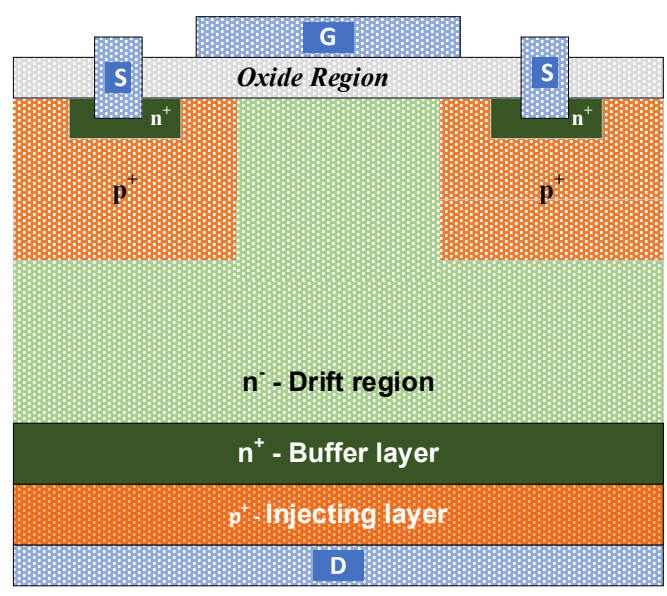

Fig. 2. (a) Representative 2-D device structure of an IGBT device.

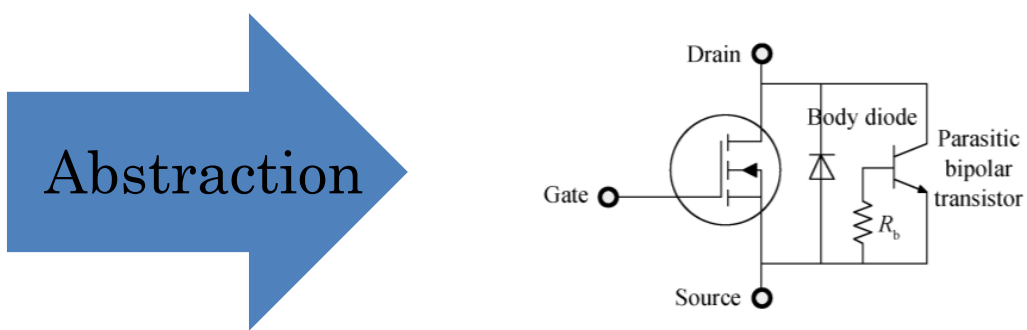

Fig. 1. (b) Equivalent circuit representation in device's large signal topology.

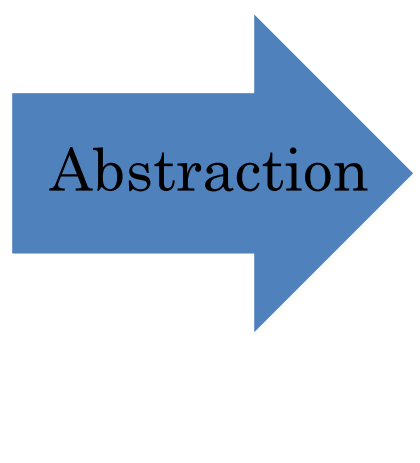

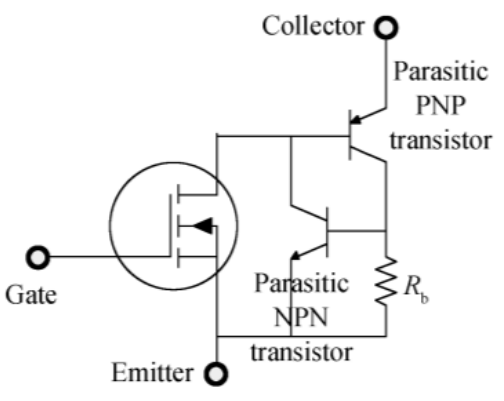

Fig. 2. (b) Equivalent circuit representation in device's large signal topology.
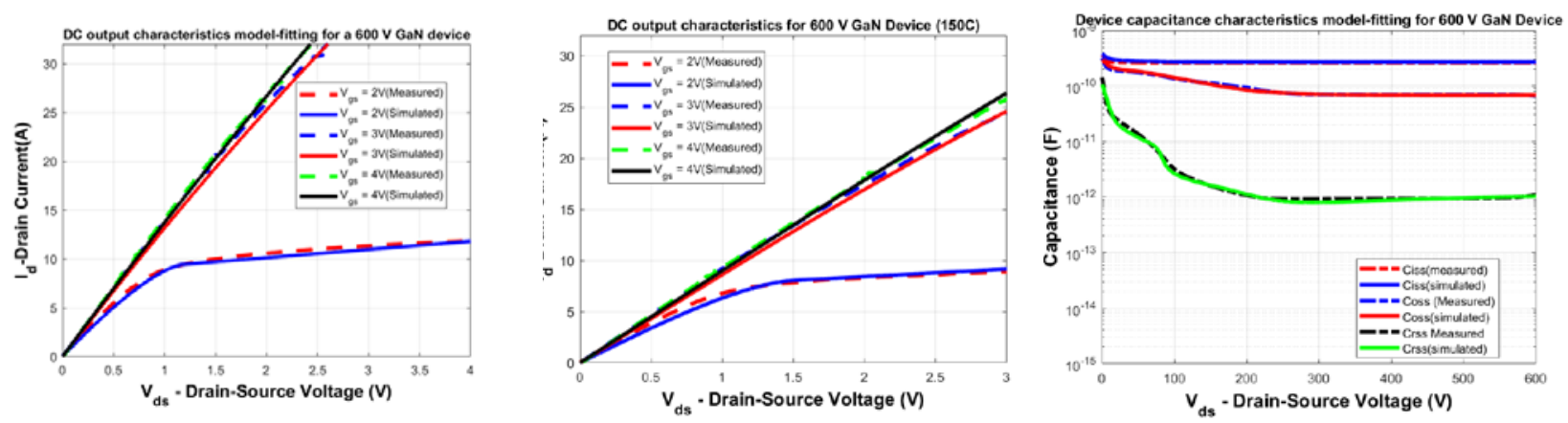

Figure 3 Model Characterization for 1-D SPICE models of GaN power devices 


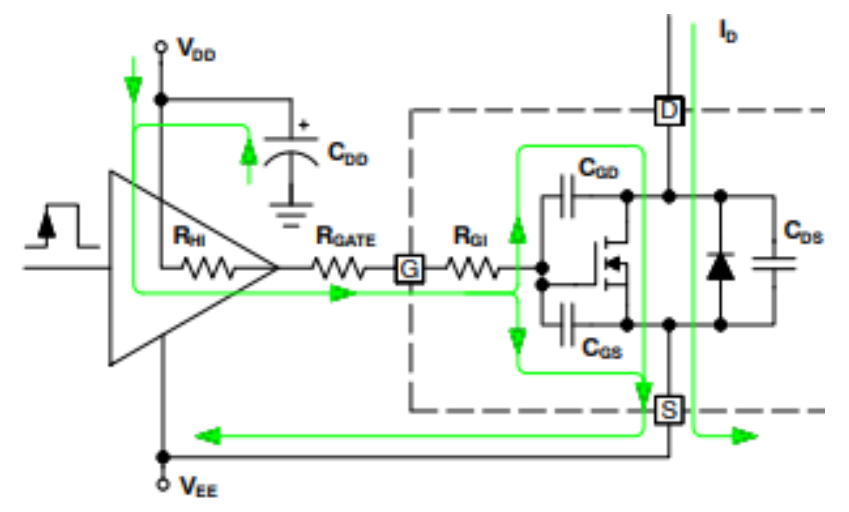

Fig. 4(a) On-state behavior of a device characterized in a switchmode circuit

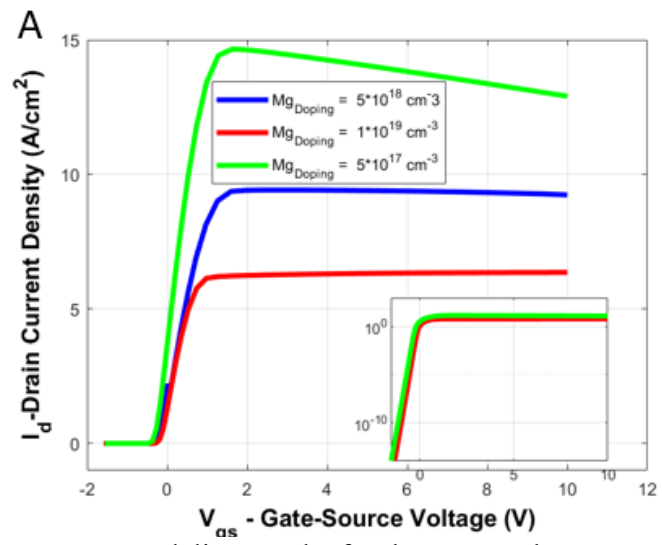

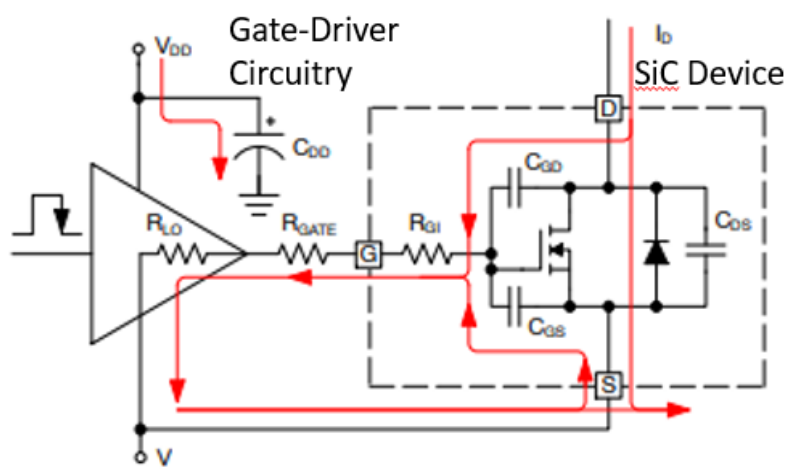

Fig. 4(b). Off-state behavior of a device characterized in a switchmode circuit

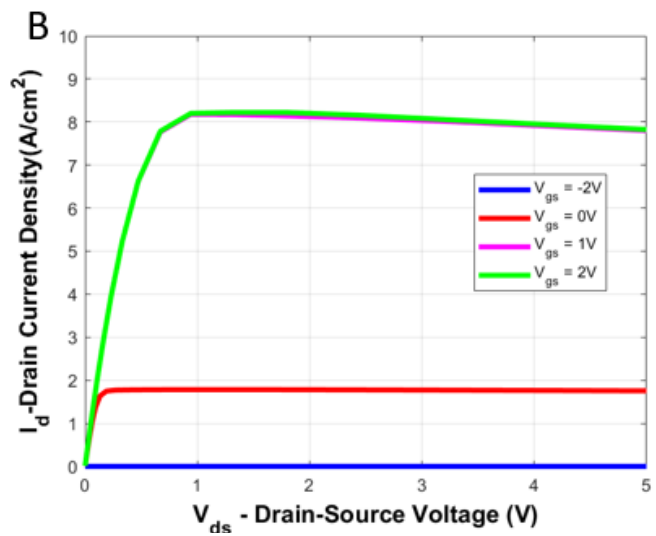

Fig. 5 TCAD Modeling results for the $\mathrm{Ga}_{2} \mathrm{O}_{3}$ planar power MOSFET device, including (a) transfer characteristics for different $\mathrm{Mg}$ doping levels, with logarithmic plot in inset, and (b) dc output characteristics for different gate voltages
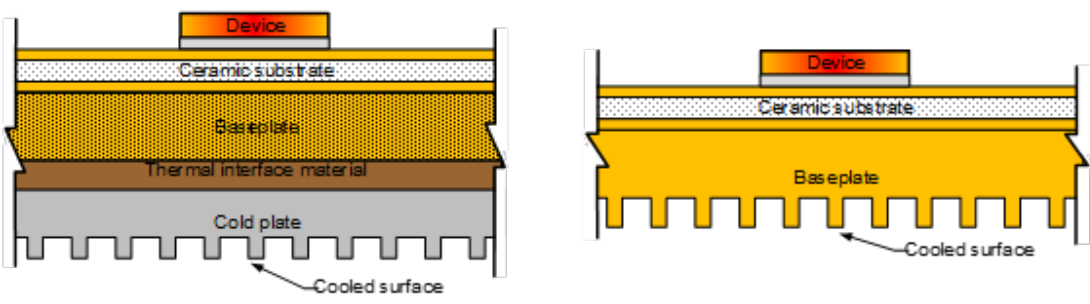

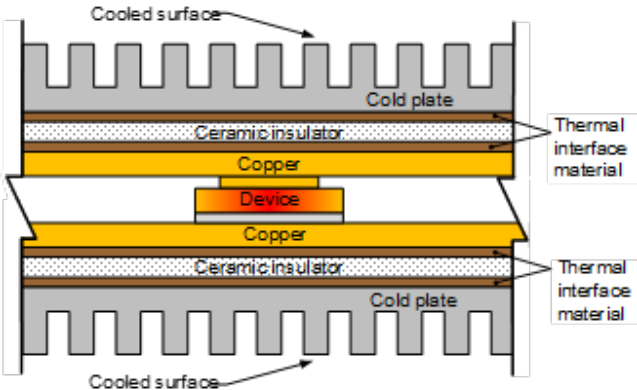

Fig. 6. Typical liquid-cooled power module configurations, cold-plate cooled (left), baseplate cooled (middle), and double-sided cooled (right)

\section{ACKNOWLEDGMENT}

This work was authored by the National Renewable Energy Laboratory (NREL), operated by Alliance for Sustainable Energy, LLC, for the U.S. Department of Energy (DOE) under Contract No. DE-AC36-08GO28308. This work was supported by the Laboratory Directed Research and Development (LDRD) Program at NREL. The views expressed in the article do not necessarily represent the views of the DOE or the U.S. Government. The U.S. Government retains and the publisher, by accepting the article for publication, acknowledges that the U.S. Government retains a nonexclusive, paid-up, irrevocable, worldwide license to publish or reproduce the published form of this work, or allow others to do so, for U.S. Government purposes. 